\title{
Unstable angina: trends and characteristics associated with length of hospitalization in the face of diminishing frequency-an evidence of a paradigm shift
}

\author{
Aaqib H. Malik ${ }^{1}$, Nauman Siddiqui ${ }^{2}$, Wilbert S. Aronow ${ }^{1}$ \\ ${ }^{1}$ Department of Medicine, Division of Cardiology, Westchester Medical Center and New York Medical College, Valhalla, NY, USA; ${ }^{2}$ Department of \\ Hematology and Oncology, Tufts Medical Center, Boston, MA, USA \\ Contributions: (I) Conception and design: AH Malik; (II) Administrative support: WS Aronow; (III) Provision of study materials or patients: AH \\ Malik, N Siddiqui; (IV) Collection and assembly of data: AH Malik, N Siddiqui; (V) Data analysis and interpretation: All authors; (VI) Manuscript \\ writing: All authors; (VII) Final approval of manuscript: All authors \\ Correspondence to: Wilbert S. Aronow, MD, FACC, FAHA. Professor of Medicine, Cardiology Division, Westchester Medical Center and New York \\ Medical College, Macy Pavilion, Room 141, Valhalla, NY 10595, USA. Email: wsaronow@aol.com.
}

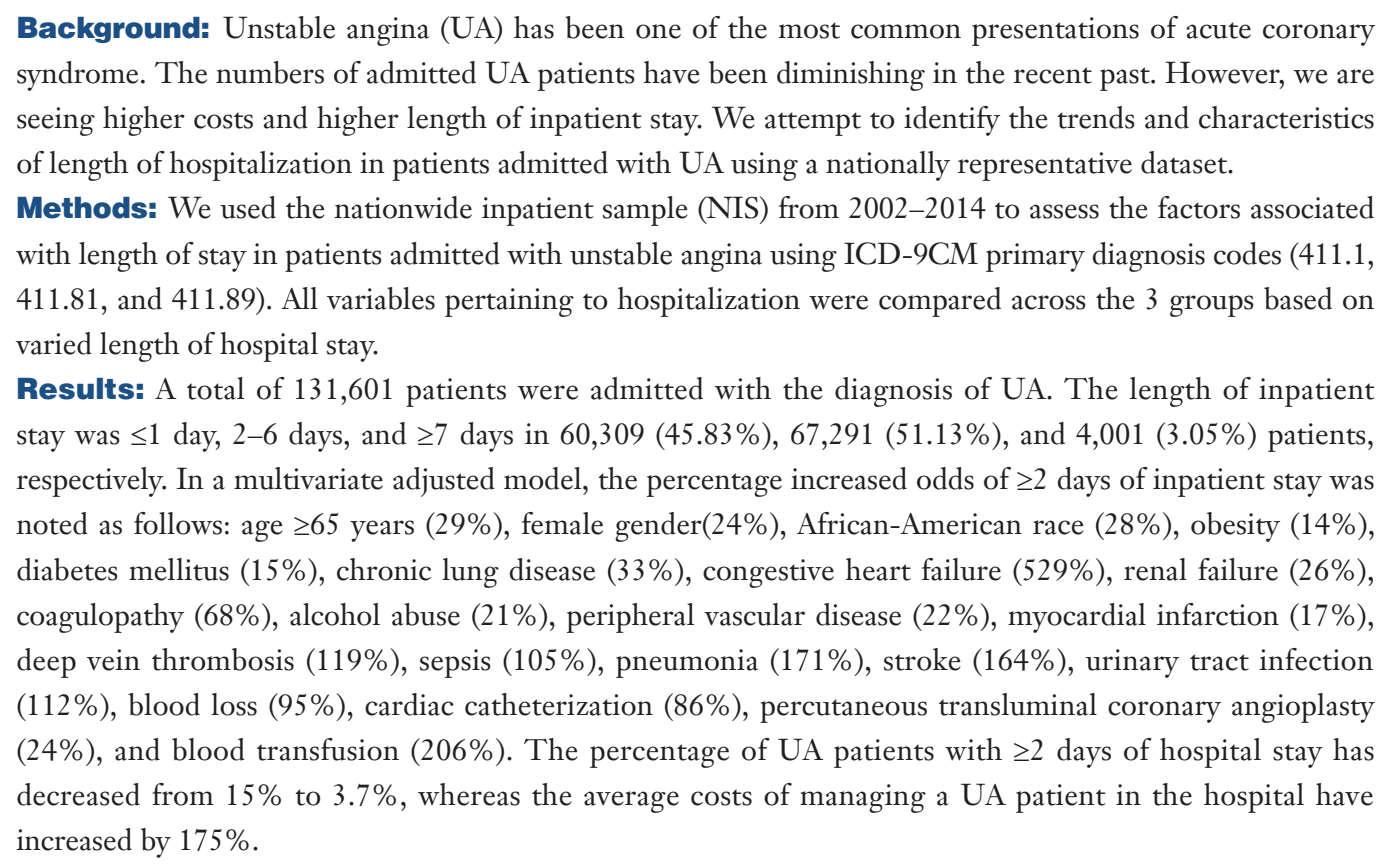

Conclusions: More than half of patients admitted with UA stay in the hospital for $\geq 2$ days, with the most important determinants being preexisting medical comorbidities and inpatient complications.

Keywords: Unstable angina; length of stay; acute coronary syndrome (ACS)

Submitted Aug 23, 2018. Accepted for publication Nov 02, 2018.

doi: $10.21037 /$ atm.2018.11.10

View this article at: http://dx.doi.org/10.21037/atm.2018.11.10 


\section{Introduction}

Unstable angina (UA) belongs to a spectrum of ischemic heart disease that is called acute coronary syndrome (ACS) or more recently non-ST-elevation acute coronary syndrome (NSTE-ACS). The other major component of NSTE-ACS is non-ST-elevation myocardial infarction (NSTEMI). The difference between UA and NSTEMI is the detection of a cardiac biomarker, as an evidence of myocardial damage and necrosis, despite the absence of electrocardiographic changes. Chest pain remains one of the most common presenting symptoms in the patients presenting with unstable angina. According to the most recent estimate, unstable angina is responsible for 550,000 hospital admissions in the United States each year (1). An earlier study by the National Center for Health statistics reported UA to be one of the most common diseases requiring admission to the hospital in the United States, leading to more than 3 million inpatient days (2).

The timing of the invasive strategy for these NSTE-ACS patients has long been debated with the 2014 American Heart Association/American College of Cardiology (AHA/ACC) guidelines eventually recommending an early invasive strategy for these patients (3). Contrary to this, a more recent meta-analysis of 10 randomized controlled trials showed no mortality benefit of an early invasive approach (4). However, almost $40 \%$ of the included patients in the study mentioned above did not have an elevated cardiac troponin. This was partly due to the fact that the identification of UA patients is an extremely difficult clinical task because of the lack of any objective data to help differentiate it from other causes. Therefore, the risk status of the included population in the aforementioned meta-analysis was somewhat minimized by the potential inclusion of UA patients. While there remains a controversy in managing the NSTEMI patients with early or delayed invasive intervention, we do need more insight into the characteristics of outcomes and factors associated with the varied length of inpatient stay in these UA patients. Generally accepted higher risk factors include prolonged angina $>20 \mathrm{~min}$, known history of coronary artery disease, hemodynamic compromise, and specific electrocardiographic changes. The length of hospitalization of these patients potentially presenting with chest pain and grouped under UA is extremely variable. The predictive demographic and clinical factors in UA patients are poorly studied. A better understanding and characterization of the variables resulting in hospitalization and dictating the length of stay is essential for their cost-effective management. Moreover, this would further pave the way to identify the optimal timing of an invasive approach in these UA or lowrisk NSTE-ACS patients. The objective of our current analysis is to identify the proportion and determinants of prolonged hospitalization in UA patients using a nationally representative database.

\section{Methods}

We utilized the nationwide inpatient sample (NIS) from 2002 to 2014 to assess the factors associated with the length of the hospital stay in the patients admitted with the diagnostic code of UA. NIS is the largest all-payer inpatient database in the United States and is a part of the Healthcare Quality and Utilization Project (HCUP), sponsored by the Agency for Healthcare Research and Quality (AHRQ). A comprehensive synopsis on NIS data is available at http:// www.hcup-us.ahrq.gov.

We used the international classification of diseases 9th Revision, Clinical Modification, (ICD-9-CM) primary diagnosis code $411.1,411.81$, and 411.89 to identify the patients with UA. All the variables pertaining to their hospitalization were compared across 3 groups, based on the number of days of their hospitalization $(\leq 1,2-6$, and $\geq 7$ days).

Study variables included were the patient's age, gender, race/ethnicity and a long list of medical comorbidities. These include hypertension, diabetes mellitus, dyslipidemia, valvular disease, atrial fibrillation, congestive heart failure, peripheral vascular disease, chronic lung disease, coagulopathy, renal failure, alcohol abuse, nicotine dependence, drug dependence, acquired immunodeficiency syndrome, deficiency anemias, rheumatoid arthritis/ connective tissue disease, blood loss, depression, hypothyroidism, liver disease, lymphoma, electrolytes deficiency, solid tumor, metastatic disease, neurological disorder, psychosis, obesity, paralysis, pulmonary circulation disease, ulcer disease, and weight loss.

ICD-9-CM secondary diagnosis codes were used to identify in-hospital complications such as pneumonia (486, 481, 482.8, and 482.3), acute kidney injury (584.9), urinary tract infection $(599.0,590.9)$, sepsis $(995.91,996.64,038$, 995.92, and 999.3), deep venous thrombosis (451.1, 451.2, $451.81,451.9,453.1,453.2,453.8$, and 453.9), pulmonary embolism (415.1), cardiogenic shock (785.51), stroke (430437), myocardial infarction (410.0-410.9), and in-hospital mortality. We also used ICD-9-CM procedure codes to 


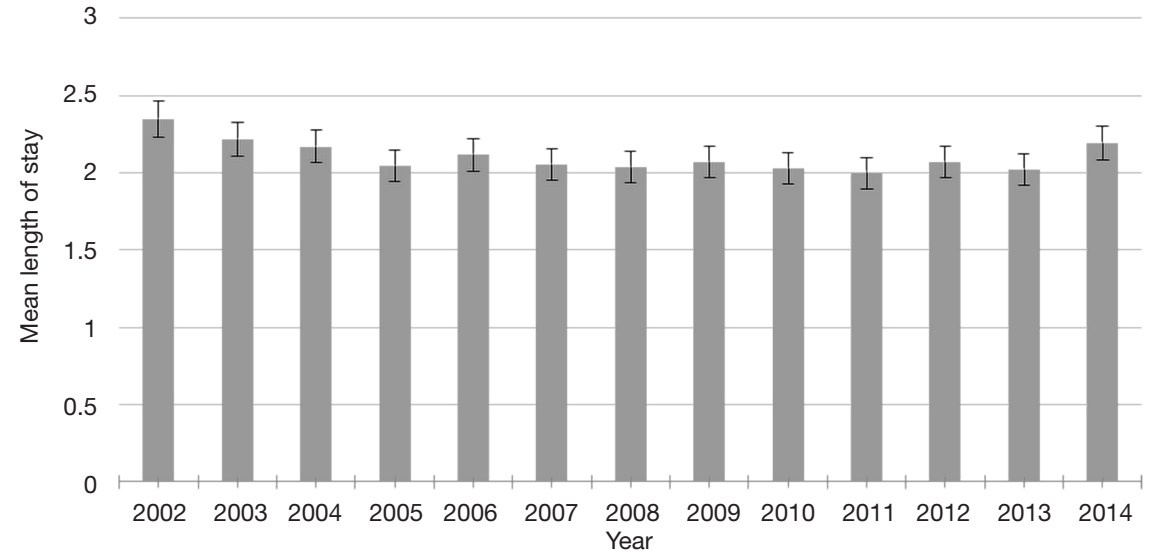

Figure 1 Mean length of hospital stay in unstable angina patients.

estimate the percentage of UA patients who underwent unstable angina related procedures such as cardiac catheterization (37.21-37.23), percutaneous transluminal coronary angioplasty (36.00-36.09, and 00.66), coronary artery bypass surgery (36.10-36.19), valvular repair (35.035.9), valve replacement (35.2), mechanical ventilation/ intubation (96.72), and blood transfusion (99.04).

Patients were also categorized based on their insurance status into Medicare, Medicaid, private/health maintenance organization, self-pay, no insurance (no charge) and other. The admitting hospitals were classified as rural, urban teaching or urban non-teaching and were also characterized by small, medium and large based on the number of hospital beds. Hospitals were looked at by their geographic location in the United States as Northeast, Midwest, West, and South.

Finally, we also looked at some other outcome data elements from NIS including total billed hospital charges, admission type (elective or non-elective, weekend or weekday) and discharge disposition. Discharge disposition was categorized into home/self-care, home health services, short-term hospital, short-term facility other including intermediate care and skilled nursing home, unknown and discharge against medical advice in the NIS data files.

\section{Statistical analysis}

The SAS 9.4 software (SAS Institute, Cary, NC) was used to convert the NIS database data to generate national estimates following the Healthcare Cost and Utilization Project (HCUP) recommendations. The weighted values of patient-level observations were generated, using pre- specified weights in the NIS to produce a nationally representative estimate of the US population of hospitalized patients. We performed descriptive and univariate analyses on demographic and clinical variables to identify the differences in study variables and endpoints across the 3 patient groups, based on their length of hospital stay. In addition, a logistic regression analysis was performed to identify the association between the patient (demographic and clinical) and hospital characteristics and the odds of "hospitalization of $\geq 2$ days". All the variables mentioned in univariate analyses were added as "predictor variables" to form a logistic regression model. These variables were retained in the final model if $\mathrm{P}$ value was $<0.1$. Finally, we performed a trend analysis to determine any change in the annual average hospitalization frequency and days of unstable angina patients from 2002 through 2014.

\section{Results}

A total of 131,601 patients were admitted with the diagnosis of UA during the 13-year study period. The overall mean length of hospitalization was 2.14 days (95\% CI, 2.13-2.15), and the overall mean age of the admitted patients with UA was 62.59 [standard error of the mean (SEM) 0.04] years. The mean length of hospitalization has slightly decreased over the last 13 years from 2.35 days (95\% CI, 2.32-2.38) in 2002 to 2.19 days $(95 \%$ CI, 2.13-2.26) in 2014 ( $\mathrm{P}<0.001$ for trend test, Figure 1). The annual percentage of patients with UA has decreased from $15.4 \%$ in 2002 to $3.7 \%$ in 2014 $(\mathrm{P}<0.0001$ for trend test, Figure 2). Over the last 13 years, the mean charges for UA admission were 15,830 (95\% CI, 15,718.60-15,941.87) dollars. 


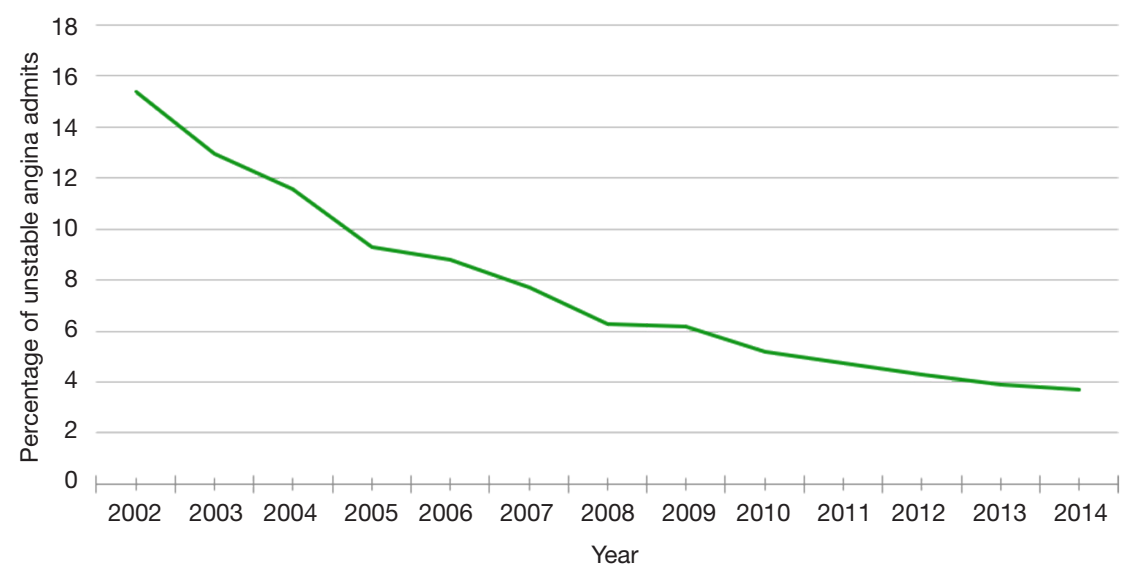

Figure 2 Annual percentage of patients admitted with unstable angina.

The length of hospitalization was $\leq 1$ day, $2-6$ days, and $\geq 7$ days in 60,309 (45.83\%), 67,291 (51.13\%), and 4,001 $(3.05 \%)$ patients, respectively (Table 1$)$. The mean age of the patients $(60.8,63.9$, and 68.2 years, $\mathrm{P}<0.0001)$ and the proportion of women $(45.75 \%, 53.91 \%$, and $54.42 \%$, $\mathrm{P}<0.0001)$ increased across the strata of the greater length of hospitalization.

Race/ethnicity analysis showed that the proportion of whites decreased across the strata of greater length of hospitalization $(72.93 \%, 65.34 \%$, and $60.31 \%, \mathrm{P}<0.0001)$, whereas the proportion of African-Americans increased with greater length of hospitalization $(11.68 \%, 17.00 \%$, and $20.57 \%, \mathrm{P}<0.0001)$ (Table 1).

The comorbid conditions that significantly increased in frequency across the 3 groups of length of hospitalization include; diabetes mellitus (24.60\%, 29.03\%, and 31.77\%, $\mathrm{P}<0.0001)$, congestive heart failure $(0.02 \%, 0.13 \%$, and $2.55 \%, \mathrm{P}<0.0001)$, valvular heart disease $(0.005 \%, 0.04 \%$, and $0.69 \%, \mathrm{P}<0.0001)$, peripheral vascular disease $(4.45 \%$, $6.48 \%$, and $11.26 \%, \mathrm{P}<0.0001)$, chronic lung disease $(14.94 \%, 19.55 \%$, and $27.14 \%, \mathrm{P}<0.0001)$, coagulopathy $(0.77 \%, 1.48 \%$, and $5.51 \%, \mathrm{P}<0.0001)$, renal failure (4.28\%, $7.85 \%$, and $18.82 \%, \mathrm{P}<0.0001)$, and alcohol abuse $(2.46 \%, 2.58 \%$, and $3.46 \%, \mathrm{P}=0.0005)$. Additionally, there were a number of other comorbid conditions that were increasingly associated with a higher inpatient stay including acquired immunodeficiency syndrome, deficiency anemia, rheumatoid arthritis/connective tissue disorder, blood loss, depression, hypothyroidism, liver disease, lymphoma, electrolyte disturbances, solid tumor, metastatic disease, neurological disorder, psychosis, paralysis, pulmonary circulation disorder, ulcer disease and weight loss. We also noted that the proportion of patients with nicotine dependence decreased across the strata of the greater length of hospitalization. As was anticipated, the rates of pneumonia, acute kidney injury, deep venous thrombosis, cardiogenic shock, stroke, urinary tract infection, sepsis, and myocardial infarction were higher among patients in the strata of the greater length of hospitalization (Table 1).

The rates of inpatient procedures were consistently higher with a longer inpatient stay such as cardiac catheterization $(14.82 \%, 23.70 \%$, and $36.34 \%, \mathrm{P}<0.0001)$, percutaneous transluminal coronary angioplasty $(2.65 \%$, $4.27 \%$, and $5.89 \%, \mathrm{P}<0.0001)$, coronary artery bypass grafting $(0.001 \%, 0.011 \%$, and $0.016 \%, \mathrm{P}<0.0001)$, valve repair $(0 \%, 0.007 \%$, and $0.96 \%)$, mechanical ventilation $(0.21 \%, 0.18 \%$, and $3.49 \%, \mathrm{P}<0.0001)$, and blood transfusion $(0.36 \%, 0.03 \%$, and $0.17 \%, \mathrm{P}<0.0001)$ (Table 1 ).

Patients with the longest length of hospital stay tended to have Medicare/Medicaid insurance and were generally admitted to a large sized urban teaching hospital located in the South geographic location. The proportion of the patients discharged to a short-term hospital (39.29\%, $22.77 \%$, and $7.91 \%, \mathrm{P}<0.0001)$ was lower across the strata of the greater length of hospitalization.

In our multivariate analysis, the following demographic factors were associated with $\geq 2$ days of length of hospitalization: age $\geq 65$ years [odds ratio $(\mathrm{OR})=1.29$; 95\% CI, 1.24-1.34; $\mathrm{P}<0.0001)$, female gender $(\mathrm{OR}=1.24$, 95\% CI, 1.20-1.27; $\mathrm{P}<0.0001)$, African- Americans race $(\mathrm{OR}=1.28 ; 95 \% \mathrm{CI}, 1.16-1.40 ; \mathrm{P}<0.0001)$, and Hispanic ethnicity (OR $=1.21 ; 95 \% \mathrm{CI}, 1.10-1.33 ; \mathrm{P}<0.0001)$ (Table 2). The following comorbid conditions were associated with the higher length of hospitalization of $\geq 2$ days; obesity 
Table 1 Clinical and demographic characteristics of unstable angina patients according to the length of hospitalization

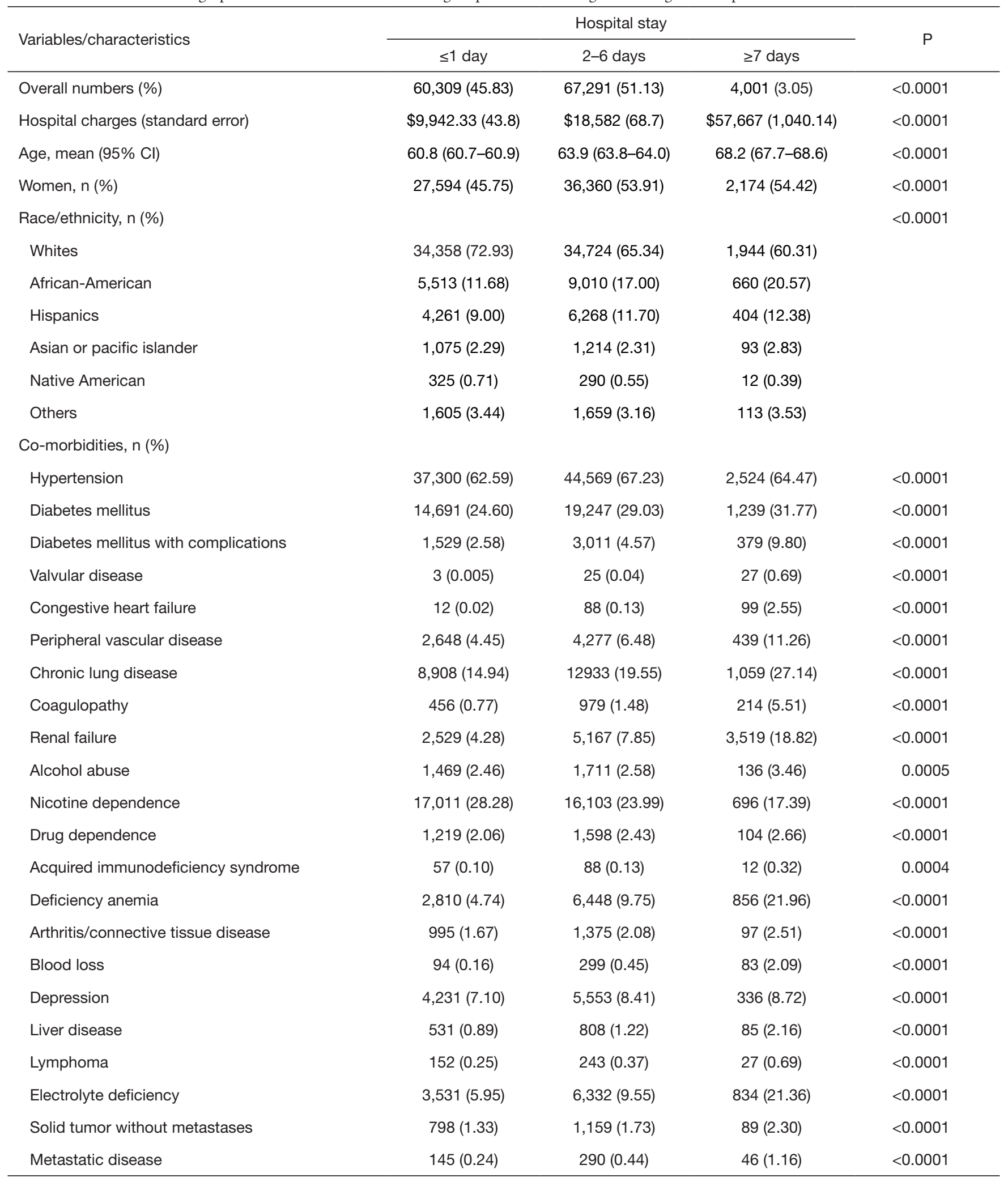

Table 1 (continued) 
Table 1 (continued)

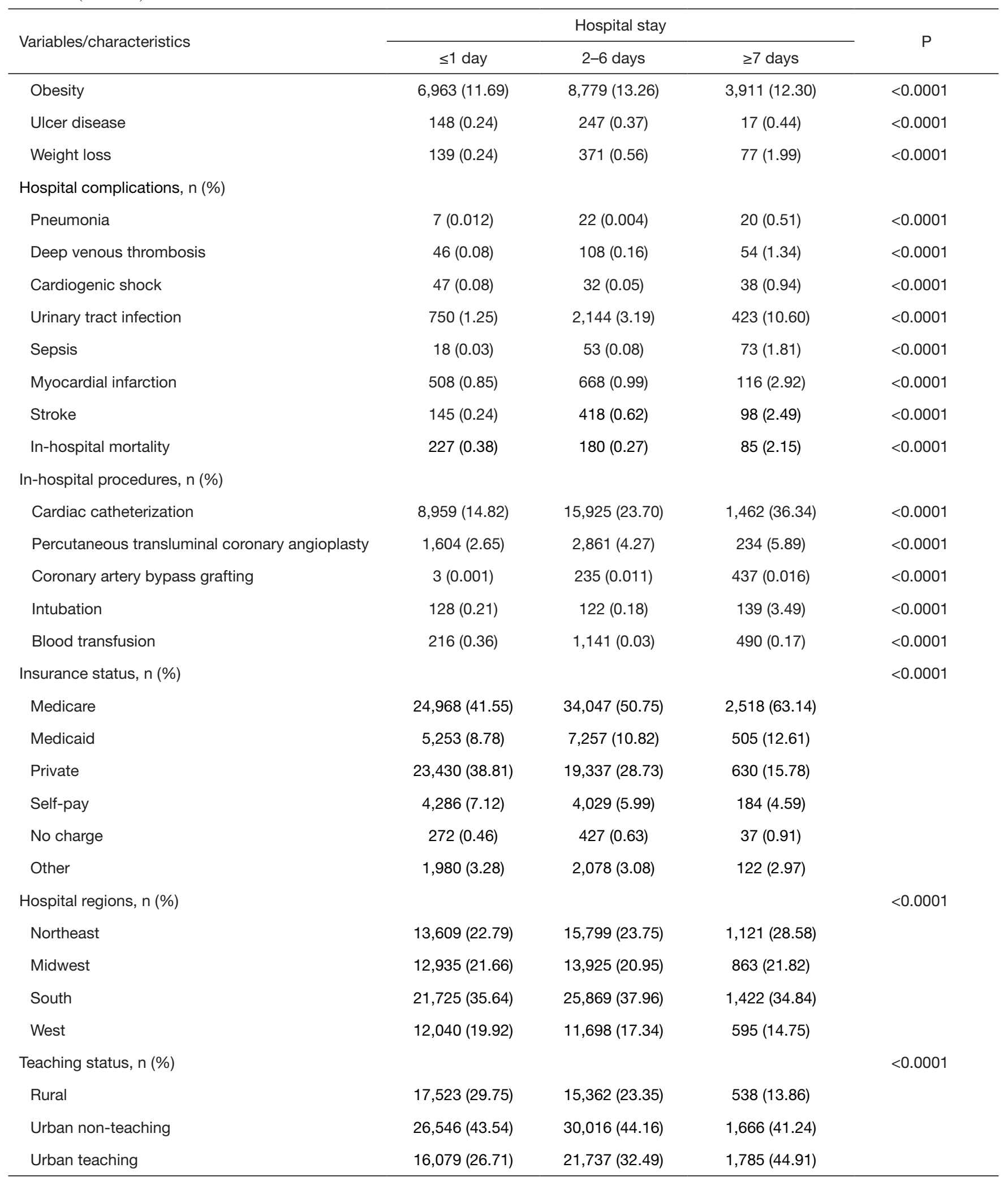

Table 1 (continued) 
Table 1 (continued)

\begin{tabular}{|c|c|c|c|c|}
\hline Variables/characteristics & \multicolumn{3}{|c|}{ Hospital stay } & $P$ \\
\hline Disposition, n (\%) & & & & $<0.0001$ \\
\hline Home/self-care & $30,929(51.14)$ & $44,412(65.97)$ & $2,122(52.87)$ & \\
\hline Short-term hospital & $23,604(39.29)$ & $15,308(22.77)$ & $315(7.91)$ & \\
\hline Home health service & $453(0.76)$ & $2,577(3.87)$ & $607(15.30)$ & \\
\hline Left against medical advice & $3,203(5.33)$ & $1,076(1.61)$ & $43(1.05)$ & \\
\hline Discharge to unknown & $21(0.03)$ & $35(0.05)$ & $3(0.07)$ & \\
\hline Weekday admission & 49,292 (81.73) & $48,945(72.77)$ & $3,286(82.04)$ & $<0.0001$ \\
\hline Medium & $17,827(29.60)$ & $19,099(28.49)$ & $1,055(26.73)$ & \\
\hline Large hospitals & $29,101(49.17)$ & $36,168(54.63)$ & $2,453(61.89)$ & \\
\hline
\end{tabular}

Table 2 Univariate and multivariate analysis of demographic and clinical factors of unstable angina patients associated with $\geq 2$ days length of inpatient stay

\begin{tabular}{|c|c|c|c|c|}
\hline Variables/characteristics & \multicolumn{2}{|c|}{ Univariate analysis } & \multicolumn{2}{|c|}{ Multivariate analysis } \\
\hline \multicolumn{5}{|l|}{ Age } \\
\hline$<65$ years & Reference & & Reference & \\
\hline$\geq 65$ years & $1.48(1.45-1.51)$ & $<0.0001$ & $1.29(1.24-1.34)$ & $<0.0001$ \\
\hline Men & Reference & & Reference & \\
\hline Women & $1.39(1.36-1.42)$ & $<0.0001$ & $1.24(1.20-1.27)$ & $<0.0001$ \\
\hline \multicolumn{5}{|l|}{ Race/ethnicity } \\
\hline Asian or Pacific islander & Reference & & Reference & \\
\hline Hispanics & $1.28(1.17-1.40)$ & $<0.0001$ & $1.21(1.10-1.33)$ & $<0.0001$ \\
\hline Native American & $0.75(0.63-0.90)$ & 0.0016 & $0.78(0.65-0.95)$ & 0.0107 \\
\hline Others & $0.90(0.81-1.00)$ & 0.0600 & $0.83(0.74-0.93)$ & 0.0014 \\
\hline \multicolumn{5}{|l|}{ Co-morbid conditions } \\
\hline Obesity & $1.15(1.11-1.19)$ & $<0.0001$ & $1.14(1.10-1.19)$ & $<0.0001$ \\
\hline Hypertension & $1.22(1.19-1.25)$ & $<0.0001$ & $1.03(1.00-1.06)$ & 0.0765 \\
\hline
\end{tabular}

Table 2 (continued) 
Table 2 (continued)

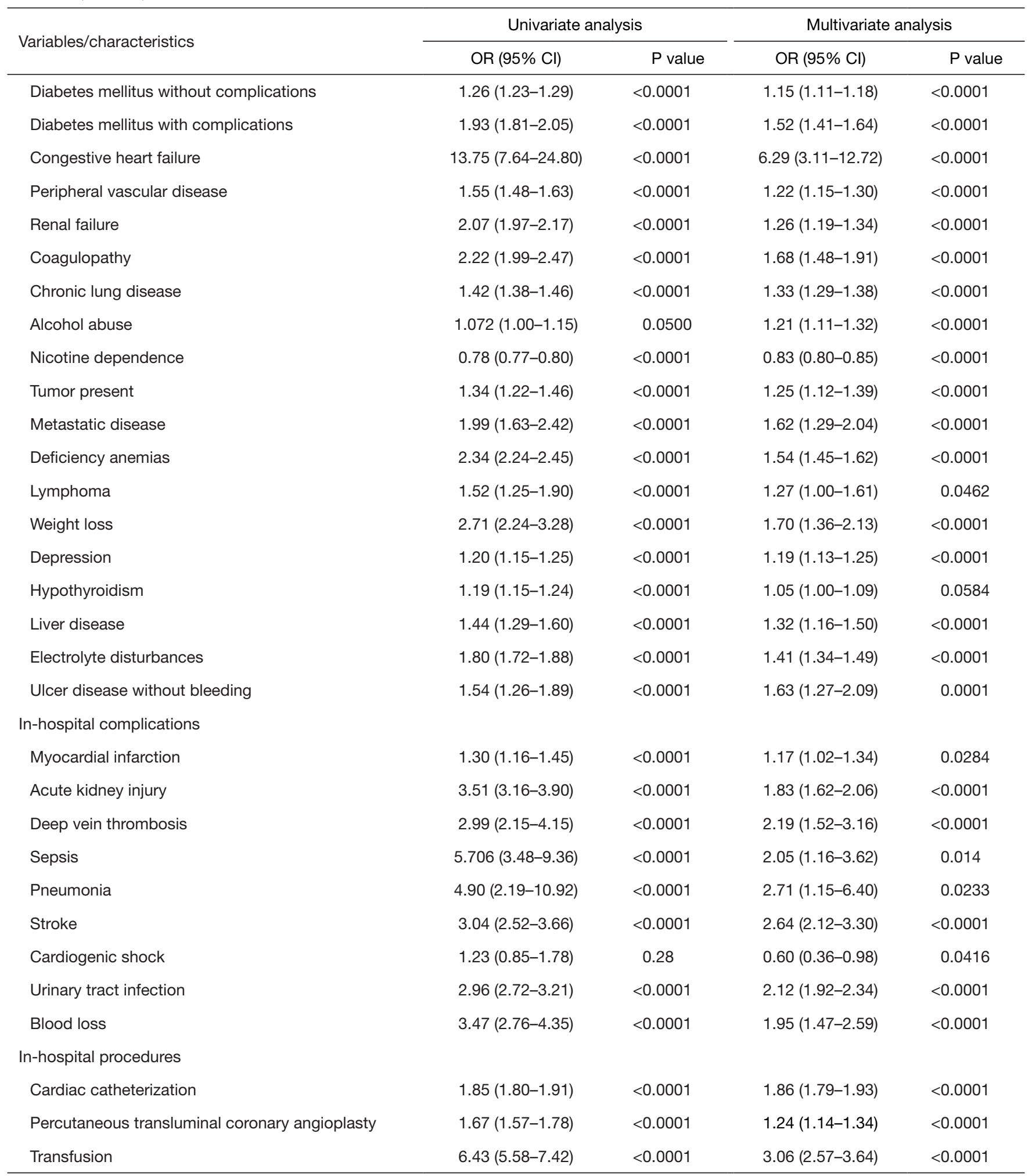

Table 2 (continued) 
Table 2 (continued)

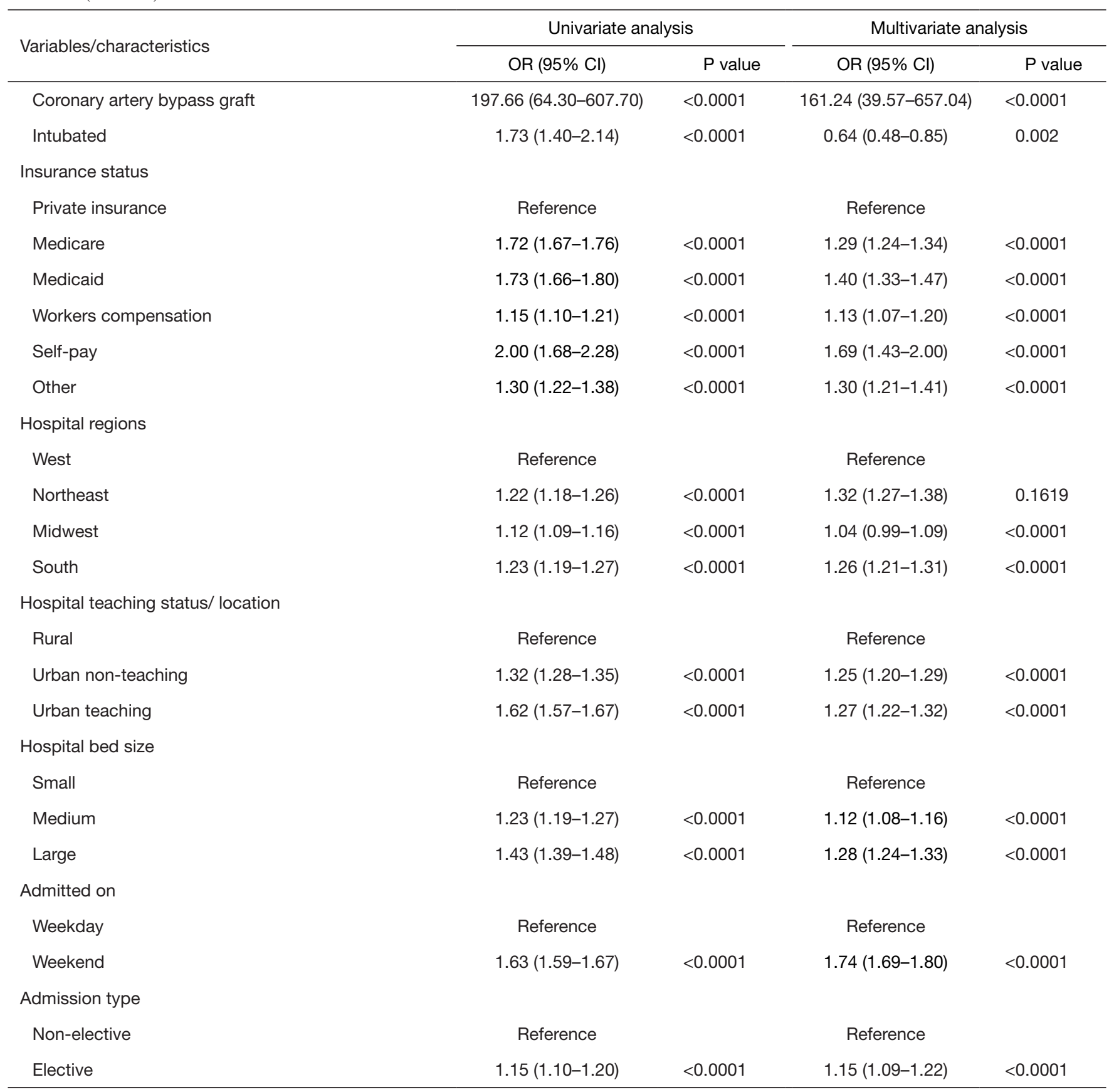

$(\mathrm{OR}=1.14 ; 95 \%$ CI, $1.10-1.19 ; \mathrm{P}<0.0001)$, diabetes mellitus (OR $=1.15 ; 95 \% \mathrm{CI}, 1.11-1.18 ; \mathrm{P}<0.0001)$, chronic lung disease $(\mathrm{OR}=1.33$; $95 \% \mathrm{CI}, 1.29-1.38 ; \mathrm{P}<0.0001)$, congestive heart failure $(\mathrm{OR}=6.29 ; 95 \% \mathrm{CI}, 3.11-12.72$; $\mathrm{P}<0.0001)$, renal failure $(\mathrm{OR}=1.26$; 95\% CI, 1.19-1.34; $\mathrm{P}<0.0001)$, coagulopathy ( $\mathrm{OR}=1.68,95 \% \mathrm{CI}, 1.48-1.91$; $\mathrm{P}<0.0001)$, alcohol abuse (OR $=1.21 ; 95 \% \mathrm{CI}, 1.11-1.32$;
$\mathrm{P}<0.0001)$, and peripheral vascular disease $(\mathrm{OR}=1.22$; $95 \%$ CI, 1.15-1.30; $\mathrm{P}<0.0001$ ) (Table 2). In addition, the other comorbid conditions significantly associated with higher length of stay in a multivariate analysis, include presence of a tumor, metastatic disease, deficiency anemia, lymphoma, weight loss, depression, liver disease, electrolyte disturbances, paralysis, psychosis, and ulcer disease. 
In-hospital complications significantly increased the odds of patient staying $\geq 2$ days in the hospital such as myocardial infarction ( $\mathrm{OR}=1.17 ; 95 \% \mathrm{CI}, 1.02-1.34 ; \mathrm{P}=0.0284)$, acute kidney injury $(\mathrm{OR}=1.83$; 95\% CI, 1.62-2.06; $\mathrm{P}<0.0001$ ), deep vein thrombosis $(\mathrm{OR}=2.19 ; 95 \% \mathrm{CI}, 1.52-3.16$; $\mathrm{P}<0.0001)$, sepsis $(\mathrm{OR}=2.05 ; 95 \% \mathrm{CI}, 1.16-3.62 ; \mathrm{P}=0.014)$, pneumonia $(\mathrm{OR}=2.71 ; 95 \% \mathrm{CI}, 1.15-6.40 ; \mathrm{P}<0.0233)$, stroke $(\mathrm{OR}=2.64 ; 95 \% \mathrm{CI}, 2.12-3.30 ; \mathrm{P}<0.0001)$, urinary tract infection $(\mathrm{OR}=2.12 ; 95 \% \mathrm{CI}, 1.92-2.34$; $\mathrm{P}<0.0001)$, and blood loss (OR $=1.95 ; 95 \%$ CI, 1.47-2.59; $\mathrm{P}<0.0001$ ) (Table 2). Similarly, in-hospital procedures were associated with length of hospitalization of $\geq 2$ days: cardiac catheterization ( $\mathrm{OR}=1.86 ; 95 \% \mathrm{CI}, 1.79-1.93 ; \mathrm{P}<0.0001)$, percutaneous transluminal coronary angioplasty $(\mathrm{OR}=1.24$; 95\% CI, 1.14-1.34; $\mathrm{P}<0.0001)$, and transfusion $(\mathrm{OR}=3.06$; 95\% CI, 2.57-3.64; $\mathrm{P}<0.0001)$ coronary artery bypass graft $(\mathrm{OR}=161.24 ;$ 95\% CI, 39.57-657.04; $\mathrm{P}<0.0001)$ (Table 2).

In addition, we noted that compared to private insurance, self-pay, Medicare, and Medicaid have the highest odds (29\%, $40 \%$, and $69 \%$ respectively) to be associated with $\geq 2$ days of length of inpatient stay in UA patients. Compared to hospitals in the Western region, UA patients in Northeastern and Southern hospitals appear to have higher odds (32\% and 26\% respectively) of longer inpatient stay. Elective admits, weekend admits, and larger urban teaching facilities had higher odds $(15 \%, 74 \%$, and $28 \%$ respectively) of having a longer inpatient stay.

\section{Discussion}

The main finding of this analysis of admitted UA patients in the United States is that an estimated $54.2 \%$ of these patients were admitted to the hospital for $\geq 2$ days. Longer inpatient stay of these UA patients was found to be associated with increased medical comorbidities, and higher in-hospital procedures and complications. The percentage of patients undergoing coronary angiography (CA) for UA ranged from $14.82 \%$ to $36.34 \%$, but approximately every 1 out of 7 patients who had CA underwent percutaneous coronary intervention (PCI). This translates into the fact that approximately $86 \%$ of the UA patients who underwent CA did not require PCI suggesting that majority of UA patients are lower risk and they do not require immediate coronary intervention. Elgendy et al. came up with similar findings in female patients with UA suggesting an early invasive strategy for the management of NSTE-ACS is not associated with improved outcomes (5). These findings suggest that UA patients, defined by the lack of release of the coronary biomarker in the blood, are inherently lowrisk NSTE-ACS as there have been numerous other studies that have shown the benefit of early invasive treatment in NSTEMI patients (6-13).

There has been a paradigm shift in the statistics of UA patients over the last 30 years stemming from upclassification of a large majority of these patients. It has always been imperative to differentiate between NSTEMI and UA since the patients with NSTEMI carry almost twice the mortality risk compared to the UA patients (14). In the late 90s, cardiac troponins replaced creatine kinasemyocardial band (CK-MB) as a primary biomarker, and that resulted in up-classification of almost $33 \%$ of UA patients into the NSTEMI cohort (15). The fraction of the UA patients is expected to plunge even further after an expected widespread implementation of the highly sensitive troponin assay. We anticipate a lot more of these UA patients would eventually be up-classified into NSTEMI, although a recent study had underwhelming results in the prognostic value of highly sensitive troponin (16).

As shown in Figure 2, there has been a consistent downward trend in the frequency of UA patients over the last several years, and, for the reasons mentioned above, it is expected to decline further. Future studies will hopefully help us characterize these diminishing percentages of UA patients into a lower risk NSTE-ACS category, which potentially could be discharged after a much shorter hospital stay with a closer outpatient follow-up. As noted above, more than half of the UA patients stay in the hospital for $\geq 2$ days. In addition to evaluation of these patients from an ischemic heart disease perspective, another reason for an increase in the length of hospitalization of these patients could be due to the days spent in medical co-management and stabilization of their comorbid conditions. The length of hospitalization also remains an important determinant of the hospitalization charges. Figure 3 shows that there has been an exponential rise in the costs associated with UA patients. This is partly explained and reflected in Figure 4, as we notice an expected trend of increasing cost of hospital stay across the increasing length of hospitalization.

Our multivariate analysis also shows that the patients who are $\geq 65$ are $29 \%$ more likely to be admitted for a longer in-hospital stay. The simplistic explanation for this would be increased co-morbidity in this cohort group. But the issue is much more complex, and we must consider social barriers such as the lack of home support, caregiver distress, and arrangement of patients' potential shortterm disposition. Racial and gender disparities in the 


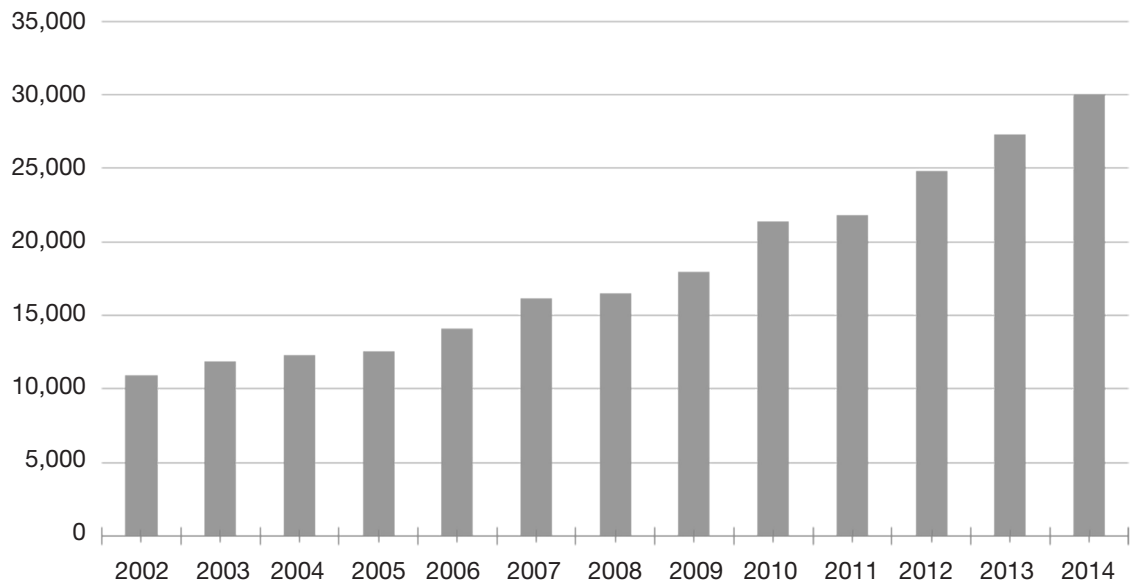

Figure 3 Annual mean hospitalization cost of unstable angina patients (\$).

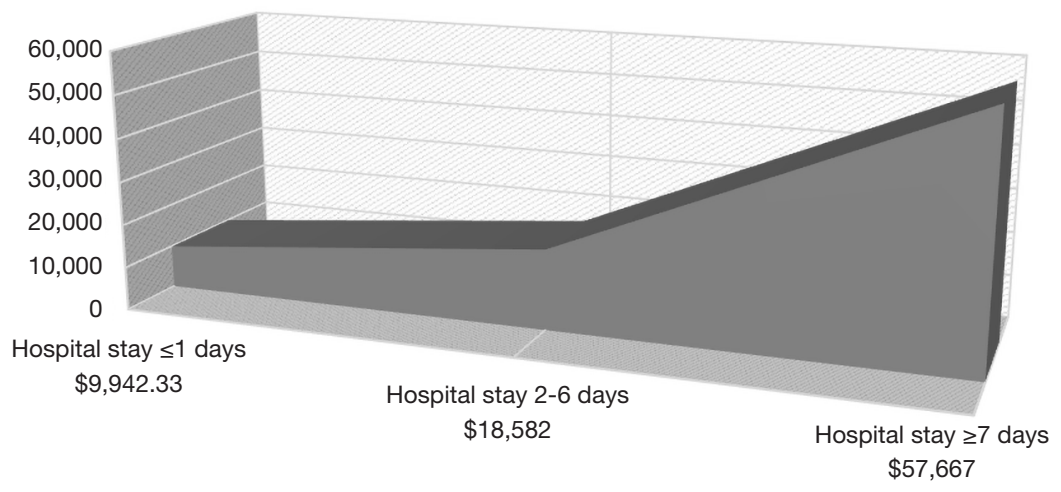

Figure 4 Mean hospital charges of unstable angina patients across the length of hospitalization.

management of UA patients exists as being an AfricanAmerican or being a woman puts you at higher odds of having a longer inpatient hospital stay (28\% and $24 \%$ respectively). This is also consistent with prior studies with different diagnoses $(17,18)$.

As was expected, the variables for a longer hospital stay in UA patients does include a variety of co-morbid conditions, in-hospital procedures, and in-hospital complications. This is also consistent with the prior ACS studies (19). Of the co-morbid conditions, the most significant of them is congestive heart failure, complicated diabetes mellitus, coagulopathy, weight loss, metastatic disease, and paralysis (Table 2). Surprisingly, nicotine dependence is associated with a lower stay in the hospital. Naturally, a longer inpatient stay leads to more inpatient complications such as pneumonia, urinary tract infection, sepsis, myocardial infarction, stroke, deep venous thrombosis and blood loss as shown in our multivariate-adjusted model. Patients with cardiogenic shock and intubations are associated with a lower stay, which possibly could be explained by an increased mortality in this group. With a longer inpatient stay, the patients underwent significantly more cardiology related procedures. As an example, patients who stayed more than 1 day in the hospital with UA were $86 \%$ more likely to undergo cardiac catheterization and $24 \%$ more likely to have an angioplasty or a coronary artery bypass graft.

There seems to be a trend in the geographic distribution with the large urban teaching hospitals in the Northeast and South admitting these UA patients much longer compared to hospitals in the West and Midwest. This could be explained by possibly a higher case mix and more complicated patients. We also noticed a weekend effect that shows that a patient with UA, when admitted on a weekend, is $74 \%$ more likely to stay in the hospital for more than 1 day. This could be explained by the lack of resources and 
staff over the weekends, covering physicians' discomfort in discharging these patients, and/or potentially lack of familiarity with their colleagues' patients.

This study draws its strengths from the use of the NIS data, which is the largest all-payer dataset that overcomes the biases seen in a single center and small regional studies. Therefore, the results can be generalized to the population of the United States. Limitations of this study must be discussed. The earlier discharge of the any patient has safety implications in the early post discharge period. A claimsbased data such as NIS lacks post-discharge information. Even though we found that $86 \%$ of the UA patients who underwent CA did not require an intervention, we do not have information that whether the patients who did not undergo intervention performed better or worse. We are unable to attribute causality due to the observational nature of this study. This nonrandomized study is prone to potential selection and ascertainment bias. These findings should be interpreted with the knowledge and caution that the exact time frame and occurrences of various comorbid conditions, procedures, and complications during the hospitalization cannot be determined from these data. The reasoning behind higher in hospital stay could not be ascertained to comorbidities as it could be due to many other confounding factors such as social factors. Additionally, higher length of stay in these patients could have been attributed to the fact that their comorbid conditions needed to be stabilized before a final investigative study or a procedure can be performed. As mentioned prior, as this study is based on NIS dataset it has inherent limitations of its dependency on the accuracy of diagnoses and procedural codes. The diagnosis of UA as the primary diagnosis or secondary diagnosis has been reported to have a positive predictive value of $73 \%$ in a previous validation study, which means that the accuracy of ICD code to identify UA patients is suboptimal and this does add to uncertainty and limitation of this study (20). Additionally, the identification of UA patients is extremely challenging due to the lack of an objective evidence of the ischemic origin of the chest pain. Moreover, NIS data lacks laboratory results and this makes the basis of the diagnosis of UA to be clinical judgement only and it does not provide any evidence of lack of cardiac enzyme elevation. Finally, we do not have any information regarding the medications from this data hence we were unable to assess the percentage of UA requiring interventions on optimal medical therapy.

In conclusion, our study provides a detailed analysis of the hospitalization of UA patients in the United States. The length of hospitalization in patients with UA is an important surrogate marker of the effectiveness of medical interventions and a critical determinant of the cost of hospitalization. With an increased adoption of the highly sensitive cardiac troponin test, we expect to tease out a lower risk or a much-decreased cohort of UA patients. As shown and predicted by our analysis, the expected numbers and frequency of these patients will continue to decrease. These lower risk UA patients could potentially be managed with a shorter inpatient stay and likely have a much lower mortality risk compared to the UA cohort from 30 years ago. More studies are needed to evaluate that risk and to identify the cohort of the lower risk UA patients to help reduce their length of hospitalization.

\section{Acknowledgements}

None.

\section{Footnote}

Conflicts of Interest: The authors have no conflicts of interest to declare.

\section{References}

1. Lloyd-Jones D, Adams R, Carnethon M, et al. Heart disease and stroke statistics--2009 update: a report from the American Heart Association Statistics Committee and Stroke Statistics Subcommittee. Circulation 2009; 119:480-6.

2. Braunwald E, Jones HR, Mark BD, et al. Diagnosing and managing unstable angina. Agency for Health Care Policy and Research. Circulation 1994;90:613-22.

3. Amsterdam EA, Wenger KN, Brindis GR, et al. 2014 AHA/ACC guideline for the management of patients with non-ST-elevation acute coronary syndromes: executive summary: a report of the American College of Cardiology/ American Heart Association Task Force on Practice Guidelines. Circulation 2014;130:2354-94.

4. Bonello L, Laine M, Puymirat E, et al. Timing of coronary invasive strategy in non-ST-segment elevation acute coronary syndromes and clinical outcomes: An updated meta-Analysis. JACC Cardiovasc Interv 2016;9:2267-76.

5. Elgendy IY, Mahmoud NA, Mansoor H, et al. Early invasive versus initial conservative strategies for women with non-ST-elevation acute coronary syndromes: A 
nationwide analysis. Am J Med 2017;130:1059-67.

6. Invasive compared with non-invasive treatment in unstable coronary-artery disease: FRISC II prospective randomised multicentre study. FRagmin and Fast Revascularisation during InStability in Coronary artery disease Investigators. Lancet 1999;354:708-15.

7. de Winter RJ, Windhausen F, Cornel HJ, et al. Early invasive versus selectively invasive management for acute coronary syndromes. N Engl J Med 2005;353: 1095-1104.

8. Rott D, Behar S, Leor J, et al. Effect on survival of acute myocardial infarction in Killip classes II or III patients undergoing invasive coronary procedures. Am J Cardiol 2001;88:618-23.

9. Morrow DA, Cannon PC, Rifai N, et al. Ability of minor elevations of troponins I and $\mathrm{T}$ to predict benefit from an early invasive strategy in patients with unstable angina and non-ST elevation myocardial infarction: results from a randomized trial. JAMA 2001;286:2405-12.

10. Fox KA, Poole-Wilson AP, Henderson AR, et al. Interventional versus conservative treatment for patients with unstable angina or non-ST-elevation myocardial infarction: the British Heart Foundation RITA 3 randomised trial. Randomized Intervention Trial of unstable Angina. Lancet 2002;360:743-51.

11. Spacek R, Widimský P, Straka Z, et al. Value of first day angiography/angioplasty in evolving Non-ST segment elevation myocardial infarction: an open multicenter randomized trial. The VINO Study. Eur Heart J 2002;23:230-8.

12. Cannon CP, Weintraub SW, Demopoulos AL, et al. Comparison of early invasive and conservative strategies in patients with unstable coronary syndromes treated with the glycoprotein IIb/IIIa inhibitor tirofiban. N Engl J Med 2001;344:1879-87.

13. Boden WE, O'Rourke AR, Crawford HM, et al. Outcomes in patients with acute non-Q-wave myocardial infarction randomly assigned to an invasive as compared with a

Cite this article as: Malik AH, Siddiqui N, Aronow WS. Unstable angina: trends and characteristics associated with length of hospitalization in the face of diminishing frequencyan evidence of a paradigm shift. Ann Transl Med 2018;6(23):454. doi: $10.21037 /$ atm.2018.11.10 conservative management strategy. Veterans Affairs NonQ-Wave Infarction Strategies in Hospital (VANQWISH) Trial Investigators. N Engl J Med 1998;338:1785-92.

14. Antman EM, Tanasijevic JM, Thompson B, et al. Cardiacspecific troponin I levels to predict the risk of mortality in patients with acute coronary syndromes. $\mathrm{N}$ Engl J Med 1996;335:1342-9.

15. Braunwald E, Antman ME, Beasley WJ, et al. ACC/ AHA guidelines for the management of patients with unstable angina and non-ST-segment elevation myocardial infarction: executive summary and recommendations. A report of the American College of Cardiology/American Heart Association task force on practice guidelines (committee on the management of patients with unstable angina). Circulation 2000;102:1193-209.

16. Magnoni M, Gallone G, Ceriotti F, et al. Prognostic implications of high-sensitivity cardiac troponin $\mathrm{T}$ assay in a real-world population with non-ST-elevation acute coronary syndrome. Int J Cardiol Heart Vasc 2018;20:14-9.

17. Bolden L, Wicks NM. Length of stay, admission types, psychiatric diagnoses, and the implications of stigma in African Americans in the nationwide inpatient sample. Issues Ment Health Nurs 2005;26:1043-59.

18. Bramkamp M, Radovanovic D, Erne P, et al. Determinants of costs and the length of stay in acute coronary syndromes: a real life analysis of more than 10,000 patients. Cardiovasc Drugs Ther 2007;21:389-98.

19. Jan S, Lee WS, Sawhney PJ, et al. Predictors of highcost hospitalization in the treatment of acute coronary syndrome in Asia: findings from EPICOR Asia. BMC Cardiovasc Disord 2018;18:139.

20. Varas-Lorenzo C, Castellsague J, Stang RM, et al. Positive predictive value of ICD-9 codes 410 and 411 in the identification of cases of acute coronary syndromes in the Saskatchewan Hospital automated database. Pharmacoepidemiol Drug Saf 2008;17:842-52. 